\title{
The Use of UAVs to Save People in the Mountains: Tasks, Problems, Perspective
}

\author{
Irina K. Romanova ${ }^{1 *}$ \\ ${ }^{1}$ Bauman Moscow State Technical University, 2nd Baumanskaya str., 5/1, 105005, Moscow, Russia
}

\begin{abstract}
The actual problem of organizing and conducting rescue operations in the mountains is considered. The achievements associated with the following factors were noted: the active development of UAVs of various designs, masses, and equipment; development of new information systems, including sensors, communication systems; the emergence of new image processing algorithms and other types of data based on artificial intelligence methods. It was emphasized that a fundamentally new achievement that opens up broad prospects is an integrated approach to the use of rescue equipment, including ground-based mobile equipment, UAVs of various types, global navigation and mobile communications, and professional rescuers. Problems were identified that are associated with the difficulties of the task, including a complex mountainous terrain, snow cover, avalanches, strong winds, low temperatures, immobility and even lack of communication with the victims. The article considers the problem of low temperatures, which leads to icing, which gives rise to an uncontrolled change in the properties of the control object - UAVs, difficulties in the functioning of control elements, and distortion of the sensors. Based on the methods of artificial intelligence, including modern methods of detecting damage, a comparative analysis of different approaches was carried out and a predominant approach was drawn, which allows a high degree of certainty to detect problems of UAV icing and timely change of control system settings.
\end{abstract}

\section{The Use of UAVs in Rescue Operations}

\subsection{The Relevance of the Task}

The problem of saving people, local residents and unprepared tourists, as well as those who are engaged in professional activities, including athletes, in general, everyone who was in the mountains voluntarily or, if necessary, in the mountains, has always been a very important and difficult to solve problem note that the first paragraph of a section or subsection is not indented.

The success of any emergency response and analysis depends on the timely receipt of data received from the scene [1]. The specifics of mountain rescue operations as part of search and rescue missions in uninhabited areas strongly depend on the time required to

* Corresponding author: irina.romanova@bmstu.ru 
search for a lost person [2]. In particular, in the framework of eliminating the consequences of an avalanche, one of the factors affecting the likelihood of surviving victims is the speed with which they are found and dig out [3]. This is due to emerging medical problems in affected people $[4,5]$. Although the activities of rescue services have a long history [6], modern information technologies and new vehicles give hope for a more successful solution of the task.

\subsection{UAV application concepts}

From the theses stated above it follows that it is important to find a target for search and rescue operations as soon as possible. Automatic object detection is important because a person cannot control multiple surveillance screens within 24 hours. In addition, the subject is often too small to be visible to the human eye on the surveillance screen. [7].

Advances in unmanned aerial vehicles have made it possible to use flying robots equipped with video surveillance systems, such as optical cameras, to assess the damage caused by natural or man-made disasters and to locate victims in the wreckage, i.e. search and rescue support [7], [3].

The drone is an effective tool carrier and saves time on rough terrain, which covers a large geographical area. It is a safe addition to human resources in high-risk environments such as an avalanche. An UAV equipped with an avalanche transceiver and an HD camera can improve the assessment of the situation and provide useful support in making decisions for hospital services and ski patrolling in the mountains [8]. The technology of unmanned aerial vehicles has attracted great attention, providing an effective solution, especially in real-time applications. This is mainly due to their ability to collect extremely high resolution (EHR) data in hard-to-reach areas and areas of limited coverage, due to their small size and speed of deployment, despite their ease of use and availability [9]. Thus, the use of UAVs instead of rescue people to search for victims provides a number of advantages, such as their high speed, maneuverability and the fact that they do not feel psychological pressure, which often leads to human errors [10].

Comparisons with manned aircraft show that UAVs can fly with lower altitude and lower speed than manned systems, which allows them to collect higher resolution data, they can operate in adverse weather and hazardous conditions and receive data autonomously, which makes UAVs optimal platform for quick response applications. Constantly available accurate information about the location of the unmanned system through measurements from satellite and inertial navigation systems and other resolving sensors is an advantage [1].

In general, new UAV technical solutions allow expanding the capabilities of monitoring and mapping the impact of natural disasters. UAVs, as a rule, are cheaper and more universal than traditional methods of remote sensing, and therefore they can be considered as a good alternative for obtaining images and other physical parameters before, during and after a natural disaster [11]. For the task of saving people, the combination of the efforts of ground rescue services and UAVs is especially relevant [6]. A specific implementation for finding people in the mountains shows the advantages of such a concept [12].

The most important is the preventive work in the mountains: monitoring the avalanche, mudflow and other conditions, timely notification and, if necessary, discharge of explosive charges used to launch controlled avalanches [13]. 


\subsection{Features of the situation in the highlands}

Highlands are characterized by a number of difficulties that may not be encountered in conventional rescue operations. This is the danger of avalanches [14], [37], [13], a complex mountainous terrain leading to the risks of possible UAV collisions with mountains [15].

In general, the mountainous environment can be assessed as harsh for agents involved in rescue operations: frost, wind eddies, blizzard, darkness, etc.). UAVs must be designed to be stable in this environment. For example, anti-icing mechanisms can be integrated into them. Thus, the specificity of work in the mountains is the presence of mountainous terrain and weather patterns [13].

\subsection{UAV requirements}

Briefly, you can characterize the mandatory requirements for UAVs as fault tolerance and safety [10].

To minimize the time required to detect a victim, some fundamental parameters must be taken into account when developing search algorithms [16]:

1. the quality of the sensory data collected by the UAV;

2. energy limitations of the UAV;

3. environmental hazard (e.g. winds, trees);

4. level of information exchange / coordination between drones.

Specific figures in view of the task of detection and rescue are as follows [17]:

- The distance to the ground when flying with the ability to track the terrain is usually within $1 \mathrm{~m}$ with accuracy.

- Navigation accuracy even in real conditions (wind speed up to $5 \mathrm{~m} / \mathrm{s}$ in the range from $-10^{\circ} \mathrm{C}$ to $25^{\circ} \mathrm{C}$ ) is usually within $2 \mathrm{~m}$.

- The achieved localization accuracy is less than $1 \mathrm{~m}$.

\subsection{UAV navigation}

The general concept of developing a navigation system is based on the integration of satellite and inertial navigation sensors (for example, inexpensive GNSS and IMU chips based on MEMS can be used as a budget solution) to provide continuously accessible accurate location information for an unmanned system [1].

Navigation problems are as follows. Some miniature drones are completely dependent on the global positioning system (GPS) for navigation. GPS is vulnerable to accidental or intentional interference that could cause it to fail. Even in a benign setting, it's not unusual for GPS to turn off within seconds or minutes. For UAVs that use GPS exclusively for navigation, this event can be disastrous. This article proposes an extended Kalman filter approach for estimating the location of a UAV when its GPS connection is lost using distance measurements between UAVs [18].

The problem of search and navigation is complicated by the lack of previous information, non-linear display between observations of sensors and the physical world and potentially non-Gaussian models of sensor noise. To solve these problems, we propose a distributed control algorithm using information-theoretical methods with particle filters to calculate optimal control inputs for coordinated localization of a multi-purpose vehicle. This method uses the structure of the probability distributions of the target state and the sensor measurements to calculate the control inputs that maneuver the UAV to make observations that minimize the expected future uncertainty of the target state. Since the method directly uses the state of the particle filter and the exact model of the sensor noise to 
calculate mutual information, it is no longer necessary to discard the information using the linear and Gaussian approximations [19].

\subsection{Information collection and image processing}

Since the task of detecting victims is central when using UAVs to save people in the mountains, the most important is the processing of the resulting video stream, which has its own characteristics in mountainous conditions.

Information is collected in the field of disaster response using robotic platforms, which are both ground-based mobile devices and unmanned aerial vehicles (UAVs). UAVs allow for remote verification and mapping. They are capable of delivering high-resolution images and often require minimal infrastructure. It is possible to use simultaneously several UAVs that are in the air, accelerating the collection of information from the accident site [20].

Image processing is currently being successfully implemented as part of data mining [21], of which it is actually a part.

Examples of the application of these technologies are given in [7], which describes a distributed deep learning system for the efficient and accurate recognition of small objects at large distances. In [3], [22] it is shown how a sequence of images of avalanche fragments captured by UAVs is processed using a pre-trained convolutional neural network (CNN) to detect discriminatory signs. A trained linear support vector machine (SVM) is integrated at the top of the $\mathrm{CNN}$ to detect objects of interest. In addition, we introduce a pre-processing method to increase the detection frequency and a post-processing method based on a hidden Markov model to increase the efficiency of classifier prediction. An HMM-based postprocessing method is also used to further improve classifier detection results [22]. The difference from conventional recognition is the adaptation of processing technologies as applied to the recognition of snow and clouds [23].

When processing images as part of the task of working in the mountains, there are a number of problems. The threshold processing mechanism used in pre-processing depends on the intensity of one pixel. Due to the loss of information caused by image resizing, the pixels associated with some objects do not pass the threshold, and therefore, objects are not detected [3]. A very high level of detail of the data obtained using unmanned aerial vehicles, for their proper use requires further processing using suitable approaches to image processing and analysis [9]. The height at which frames are taken is also an important factor affecting Prediction Efficiency, and the results obtained with other test videos show that garbage scanning at a lower height is preferred for better detection performance. Finally, the choice of resolution for detection should be made in accordance with the tradeoff between accuracy and time [3]. A special approach is also required to overcome the following limitations: the data sets used for training / testing are not yet fully representative. The solution here is the application of data mining methods [24].

The question also arises of where it is more efficient to process information. In [22], two operational scenarios are proposed. In the first case, data is transmitted in real time to a ground station, where processing is performed to warn the operator about the detection of objects of interest, while UAVs (or a swarm of UAVs) scan avalanche areas. In this case, the problems of communication lines between the UAV and the ground station must be solved in advance. In the second scenario, the processing is performed on board the UAV. This allows us to significantly reduce the amount of information sent to the ground station, which in this case can be reduced to simple flag information when a frame containing objects of interest is detected. The disadvantage is the processing capabilities, which are reduced compared with the capabilities of the ground station. 


\subsection{Rescue Operations Group Management}

Group control of robots is a modern and quite effective means of solving various tactical tasks, including rescue operations. Search and rescue missions can be effectively and automatically performed by small, highly maneuverable groups of unmanned aerial vehicles (UAVs) [19].

The concept of group control with several UAVs for the localization of avalanche victims is described in an optimal way in [10]. The use of UAVs instead of rescue people to search for victims provides a number of advantages, such as their high speed, maneuverability, and reduced human impact. In addition, the use of multiple UAVs allows you to complete missions regardless of the scale of the disaster due to the scalability of multi-agent systems. Reliability is provided due to redundancy: if the UAV fails, the rest of the system continues to work. Finally, the algorithm divides the victim detection time by the number of drones operating, and this property is of prime importance when large avalanches occur. In addition to the obvious benefits of using task separation, the synthesis of information is equally important. Distributed deep training can be implemented on several UAVs, considering the video streams from several UAVs as one giant image. The combination of UAV processing using sensors and image processing can help optimize UAV flight parameters, including UAV position, energy restrictions, environmental hazards, and data sharing restrictions [7]. The algorithmic implementation of the concept is given in [25]: behaviors are implemented in simulations on swarms ranging in size from 1 to 20 robots. The simulation uses actual location data, including satellite images after a natural disaster, the actual locations of damaged and flooded buildings, and realistic locations of victims based on personal surveys and accounts. The results demonstrate the value of using behavior-based swarm algorithms to control autonomous unmanned aerial vehicles for search and assessment after a natural disaster. Despite the automation of processes, the participation of experts, i.e. One approach to using several robots is semiautonomous work, controlled by a small number of specialists, and human experts are only required to make important decisions [20]. In practice, a prerequisite is real-time operation [20].

There are also problems in group management, in particular, the problem of joint planning of the actions of robots operating in the conditions of an uncertain workflow of search and rescue operations [20]. Of course, the complexity of the problem increases with cooperation with different robots. There is no difference in this setting, the actions of robots of different types should be planned taking into account the dependencies between their actions in conditions of uncertainty. Today, research on collaboration between several robots is usually focused on well-known settings, where possible robot actions are defined as a set of tasks. However, in most real-world conditions, there is a significant degree of uncertainty.

Group control of robots in the considered problem is based on the theory of multiagency. Each agent will create its own virtual model based on its history, constantly updated with new knowledge during missions [10]. You can track many aspects, such as average speed, cornering performance, etc. This knowledge should be combined with the knowledge gained by the agent during the mission in order to take into account his special conditions (for example, weather).

The actively developed theory of control of heterogeneous (heterogeneous) robots can be successfully applied in the problem under consideration. Each agent should be aware of the capabilities of other agents to solve the problem of joint coverage [10]. Agents with different sensors can interact at the same time by combining sensors, which will also make the measured data more uniform. The heterogeneity of the perceived data is due to the fact that the agent can receive different data in the same place, depending on its orientation or vertical vibrations; In addition, two agents may receive different data in the same place due 
to different sensor calibrations. Combining this data using sensory synthesis will improve consistency. On the other hand, a height adjuster can be implemented to keep the distance from the ground as stable as possible.

\subsection{Group Management Security Issues}

The most important problem is collision avoidance [26]. In [27], the safety of clear definitions of UAV group control was evaluated in an analytical way that is characteristic of the dynamics and concept of operations. Data was collected and processed, including for operations with low altitudes. Collision avoidance is usually based on two components: collision detection using sensors or algorithms and a protocol to determine what participating agents should do to prevent this [10]. Collision avoidance in this case should be aimed at as little as possible to interfere with the mission. Since navigation and organization of joint actions is impossible without the organization of information exchange, it is important to understand the features of networks with UAVs in order to ensure the possibility of reliable and safe inclusion of several coordinated UAVs in air traffic [28].

\subsection{Communications}

Security is the most important, but not the only communication and informational task. The traditional means of communication used in rescue operations are beacons from a mobile phone, using communication means for two situations: when the distance to the source of the alarm signals is known and when the distance is unknown. In [2], the possibility of simultaneous use of UAVs participating in the search mission to support voice communication between the search and rescue team in the field using Wi-Fi technology was considered. The architecture of a network model for connecting mobile subscribers was presented, which is organized on the basis of a flying network in which IEEE 802 technology.

Implementing next-generation network architectures will require the creation of new network paradigms, such as aerial WSDNs, and improvements to established theoretical foundations, such as wireless sensor networks. In addition, it is necessary to design reliable and efficient networks of unmanned aerial vehicles in order to minimize the loss of people and property [29].

Network management is described in [30], which presents the achievements in the field of unmanned aerial vehicles (UAVs) for the first network response to disaster management and discusses the issues of forecasting, assessment and response in case of disasters, as well as network architectures for geophysical, climatic and meteorological disasters, based on the interaction between UAVs and wireless communications and the sensor network. Special conditions for the implementation of communication technologies in harsh climates are shown in [31].

\subsection{Communication problems}

The organization of communication is accompanied by a number of difficulties. In Arctic satellite communications, broadband, radio coverage and other communications are limited due to the remoteness and lack of appropriate infrastructure. A recent report by the Arctic Economic Council on Broadband (2017) states that modern communications satellites operating in the Earth's geostationary orbit do not fully cover the Arctic region. Satellitebased broadband in the Arctic may experience delays, and bandwidth tends to be limited and expensive throughout the region. Low temperatures also contribute to the short season 
of building the infrastructure needed for broadband technologies, and the large amount of snow and ice makes maintenance and development of broadband technologies difficult.

\subsection{Technical Implementations}

The algorithm for capturing and processing thermal images from an unmanned aerial vehicle (UAV) and its hardware implementation are described in [32]. The system consists of two parts: an "air" scene installed in DJI Phantom 3 Standard. And the other is programmed into the receiving PC called "ground." The "air" system consists of a Flir Lepton thermal camera, a Raspberry Pi card, and a GPS module (for geolocation). After that, the images are sent to the PC via a special network. The PC performs information analysis using histograms and border detection (Canny algorithm). An algorithm has been obtained for distinguishing photographs with distinct heating points.

In [33], the general principles for constructing an onboard control complex are described, including the hardware implementation of the automation of the control system was implemented in the platform of the Arduino and Raspberry Pi microcontroller. In addition, the article presents the most common and promising way to ensure uninterrupted and reliable communication of the command post with UAVs, as well as ways to counter the considered and emergency situations.

A typical appearance of a multi-purpose UAV for mountain rescue operations is presented in [34], [17]. A multi-rotor flying platform and integrated avionics are designed to meet the environmental requirements of mountainous areas, such as low temperatures, high altitude and strong winds, providing the ability to carry various payloads (individually or together), such as: avalanche beacon (ARTVA)) with automatic signal recognition and trajectory algorithms for quick detection of snowy bodies; camera (visible and thermal) for search and rescue of missing persons in the snow and in the forest day or night; deployment of a payload to dump emergency kits or a special explosive cartridge for a controlled avalanche. The resulting small (less than $5 \mathrm{~kg}$ ) UAV is capable of performing a fully autonomous flight (including takeoff and landing) of a pre-programmed or easily customizable user task. In addition, the autopilot controls sensor measurements (i.e., beacons or cameras) to automatically update the flight mission in flight. Special features, such as terrain tracking, have been developed and implemented. UAV ground station programming is not required, with the exception of mandatory monitoring, since the rescue mission can be carried out in fully automatic mode.

Important mandatory optimal combination of technical means and information processing. [35] presented a combination of real-time UAV technology and computer vision with deep learning techniques for search and rescue. A new rescue platform has been integrated to instantly locate and save people at risk.

Features of the use of rotary UAVs are shown in [36]. In particular, the possibility of using it for delivering light loads and as a flying platform for audiovisual equipment and sensors in pre-hospital conditions, despite its limited carrying capacity and durability in flight, was noted. It is also possible to shoot high-resolution aerial video from an unmanned aerial vehicle with remote control and transmit this video wirelessly in real time. Search for avalanche beacon and remote aerial photography with RPA. Incident teams, rescuers, and emergency focal points can use this additional visual information to assess the situation, make decisions, and exchange information in major incidents.

The types of UAVs used depend on the specific task. For example, unmanned aerial vehicles with a hexagonal wing can fly faster and carry a large payload, but when they do, they should be deployed with higher safety measures (safety pilots are required for light 
aircraft). On the other hand, small unmanned aerial vehicles with a rotary wing are more mobile and can approach and provide images about objects on the ground [20].

In [4], based on the observations made during flight tests, the expediency of replacing the multirotor with a larger glider and a payload base station was noted so that it could carry a heavier payload. The multi-rotor can also be upgraded with a new flight controller for better flight stabilization with the addition of $4 \mathrm{G}$ control and management.

Depending on the specification of each UAV, some methods may be well suited for some UAVs, but not suitable for others [10]. For example, a heavy UAV has greater inertia, which changes its dynamics and, for example, reduces its ability to perform narrow turns. Therefore, it can perform large turns and then return to the second phase to cover the remaining surface due to these large turns. The use of group management in this case opens up great opportunities. The digital twin [10] can be used to optimize route planning and missions in general. Each agent will create its own virtual model based on its story, constantly updated with new knowledge during missions. Many aspects can be monitored, such as average speed, cornering performance, etc. This knowledge should be combined with the knowledge gained by the agent during the mission in order to take into account his special conditions (for example, weather), so digital twins can dynamically adapt mission of any UAV (without hard models). They can adapt to any context and event. For example, in the event of a rotor breakdown during a mission, the digital twins can react by adjusting their forecast to cover the area, in terms of time or power. This technology will obviously use an order of magnitude longer processing and storage times. The coating problem was reduced to two measurements, which makes it easier, but less accurate, because UAVs are difficult to maintain relative height with a flat surface.

\subsection{Part 1 Summary}

Despite the extensive material on concepts, algorithmic support and technical implementation, more attention is required to research and ways to overcome the problems posed by the specifics of the environment in which UAVs operate as part of rescue in the mountains, including at low temperatures, strong wind loads and difficult terrain. In this article, consider one of the aspects, namely, icing problems and their forecasting based on data mining methods.

\section{Methods}

\subsection{UAV navigation and stabilization model}

The UAV navigation and stabilization model is based on a system of equations of spatial motion [38].

$$
\begin{aligned}
& y_{y c \kappa, . x}=\dot{u}+q w-r v+g \sin \theta+\eta_{y c \kappa, x} \\
& y_{y c \kappa, y}=\dot{v}+r u-p w-g \cos \theta \sin \varphi+\eta_{y c \kappa, y} \\
& y_{y c \kappa ., z}=\dot{w}+p v-q u-g \cos \theta \cos \varphi+\eta_{y c \kappa ., z}
\end{aligned}
$$




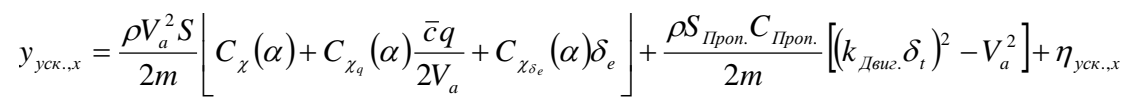

$$
\begin{aligned}
& y_{y c \kappa, y}=\frac{\rho V_{a}^{2} S}{2 m}\left[C_{Y_{0}}+C_{Y_{\beta}} \beta+C_{Y_{p}} \frac{b p}{2 V_{a}}+C_{Y_{r}} \frac{b r}{2 V_{a}}+C_{Y_{\delta_{a}}} \delta_{a}+C_{Y_{\delta_{r}}} \delta_{r}\right]+\eta_{y c \kappa, y} \\
& y_{y c \kappa, z}=\frac{\rho V_{a}^{2} S}{2 m}\left[C_{Z}(\alpha)+C_{Z_{q}}(\alpha) \frac{\bar{c} q}{2 V_{a}}+C_{Z_{\delta_{e}}}(\alpha) \delta_{e}\right]+\eta_{y c \kappa, . z}
\end{aligned}
$$

Here, the control signals are transmitted to the controls with the angles of the elevator, ailerons, rudder and throttle (angular traction): $\delta_{e} \delta_{a}, \delta_{r}, \delta_{t}$.

The model of the navigation system in Simulink for the case of longitudinal movement is presented in Fig. 1.

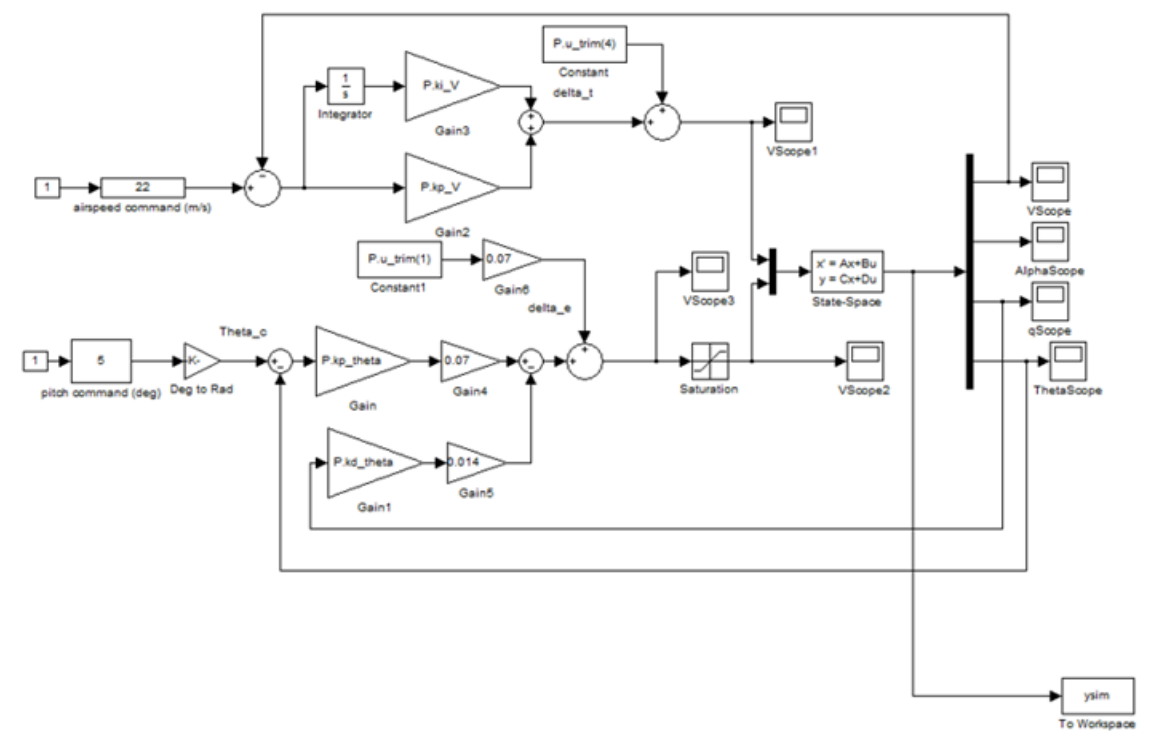

Fig. 1. Simulink navigation system model for longitudinal movement.

The model of the stabilization system for the considered case of longitudinal motion is written as follows [39]:

$$
\begin{aligned}
& \frac{d \Delta V}{d t}=a_{11 m} \Delta V+a_{12 m} \Delta \alpha+a_{13 m} \Delta \Theta+a_{14 m} \Delta \omega_{z}+b_{11 m} \Delta \delta_{e} \\
& \frac{d \Delta \alpha}{d t}=a_{21 m} \Delta V+a_{22 m} \Delta \alpha+a_{23 m} \Delta \Theta+a_{24 m} \Delta \omega_{z}+b_{21 m} \Delta \delta_{e} \\
& \frac{d \Delta \Theta}{d t}=a_{31 m} \Delta V+a_{32 m} \Delta \alpha+a_{33 m} \Delta \Theta+a_{34 m} \Delta \omega_{z}+b_{31 m} \Delta \delta_{e} \\
& \frac{d \Delta \omega_{z}}{d t}=a_{41 m} \Delta V+a_{42 m} \Delta \alpha+a_{43 m} \Delta \Theta+a_{44 m} \Delta \omega_{z}+b_{41 m} \Delta \delta_{e}
\end{aligned}
$$

or

$$
\begin{aligned}
& X^{\prime}=A X+B U ; \\
& U=\left[\begin{array}{llll}
\Delta \delta_{e}
\end{array}\right] ; \\
& X=\left[\begin{array}{llll}
\Delta V & \Delta \alpha & \Delta \Theta & \Delta \omega_{z}
\end{array}\right]^{\prime}
\end{aligned}
$$




\subsection{Models of disturbing factors}

The model of disturbances due to wind loads uses a simulation of Gaussian white noise for wind (according to Kalman) [39]:

$$
\begin{aligned}
& X^{\prime}=A X+B u+F_{b} \\
& u=\left[\Delta \delta_{e}\right],
\end{aligned}
$$

where

$$
F_{6}=W_{0} \dot{w}=\left[\begin{array}{cc}
-\cos \vartheta_{0} & -\sin \vartheta_{0} \\
-\frac{\sin \vartheta_{0}}{V_{0} c_{y}^{\alpha}} & \frac{\cos \vartheta_{0}}{V_{0} c_{y}^{\alpha}} \\
0 & 0 \\
0 & 0
\end{array} \mid\left[\begin{array}{l}
\dot{w}_{x} \\
\dot{w}_{y}
\end{array}\right]\right.
$$

And $\dot{w}_{x}, \dot{w}_{y}$ - are the components of wind loads.

The model taking into account the noise of observations in the simplest form can be written as follows:

$$
y(t)=X(t)+v(t)
$$

where $v(t)$ is the noise of observations.

In full form of the state space [39], the equations of state of observations are written as follows:

$$
x^{\bullet}(t)=A(t) x(t)+B(t) u(t)+G(t) w(t)
$$

The equations of observations are written as follows:

$$
y_{v}(t)=C(t) x(t)+D(t) u(t)+H(t) w(t)+v(t) .
$$

The model of the stabilization system with allowance for disturbances in Simulink is shown in Fig. 2. 


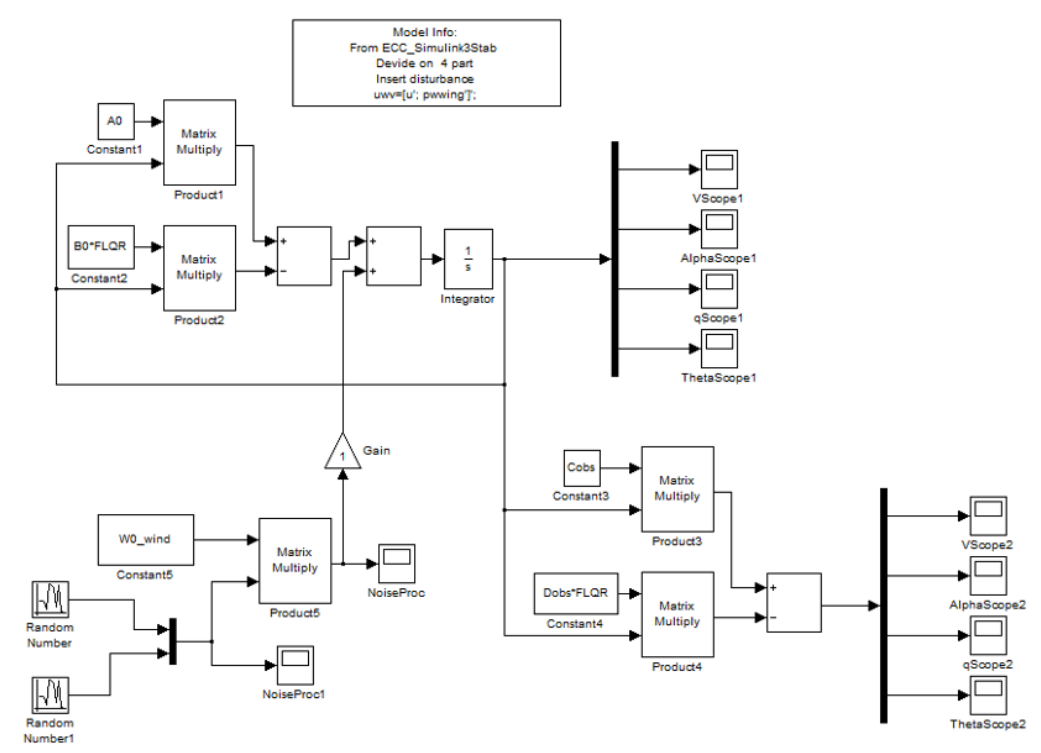

Fig. 2. Disturbance model of a stabilization system in Simulink.

\subsection{Icing disturbance model}

Icing causes changes in the property matrices of object $\mathrm{A}$, control properties $\mathrm{B}$, and observation properties $\mathrm{C}$.

The icing factor for all of the listed cases may not be the same, therefore, the coefficients of change of these matrices from the nominal values are introduced, namely:

$$
\begin{aligned}
A_{i w v} & =A \times(1+N u b) \\
B_{i w v} & =B \times(1+N u b) \\
C_{i w v} & =C \times\left[\begin{array}{cccc}
1+N u s \cdot \operatorname{signV} & 0 & 0 & 0 \\
0 & 1+N u s \cdot \operatorname{signAlf} & 0 & 0 \\
0 & 0 & 1 & 0 \\
0 & 0 & 0 & 1
\end{array}\right]
\end{aligned}
$$

The model in Simulink is shown in Fig. 3. 


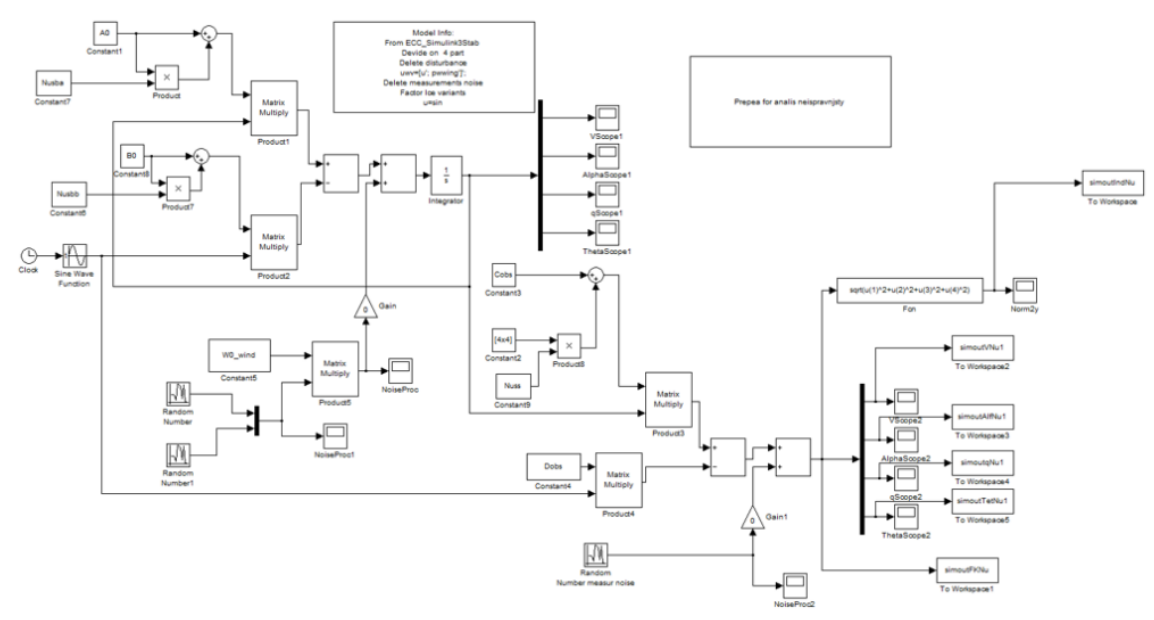

Fig. 3. Simulink model based on the effects of icing factors.

\subsection{Methods of information processing and evaluation of models and processes based on data mining}

General aspects of the use of data mining methods are described in the author [38].

In this paper, we use a new approach implemented in the MATLAB package, namely, the Predictive Maintenance Toolbox ${ }^{\circledR}[40]$.

State indicators are selected - signs of system data whose behavior changes in a predictable way as the system degrades or operates differently in operational modes. The status indicator can be any function that distinguishes normal from faulty activity or can be used to predict the remaining period of normal operation.

As state indicators in this article, simple functions of the time domain are used: average value of a specific signal (mean), variance (Signal variance), median absolute deviation (Signal Median Absolute Deviation) and others.

A sample of all possible combinations of all three factors is generated - icing results. The technology of parallel computing in MATLAB is used. A data ensemble is created to manage the simulation results. In accordance with the principles of data mining, they are divided into training and test.

\section{Results}

\subsection{Models}

The stabilization system model in MATLAB in the state space is represented by the following values of the four main matrices.

$$
\begin{aligned}
& A=x 1 \times 2 \times 3 \quad x 4 \\
& \begin{array}{llllll}
x 1 & -0.4365 & -0.8758 & -2.303 & -9.8
\end{array} \\
& \begin{array}{lllll}
x 2 & -0.02363 & -2.128 & 1.007 & 0
\end{array} \\
& \begin{array}{lllll}
x 3 & -0.1134 & -12.06 & -0.4602 & 0
\end{array} \\
& \begin{array}{lllll}
x 4 & 0 & 0 & 1 & 0
\end{array} \\
& B=u 1 u 2 \\
& \text { x1 } 59.06-0.4939 \\
& \begin{array}{lll}
x 2 & 0 & 0.2161
\end{array}
\end{aligned}
$$




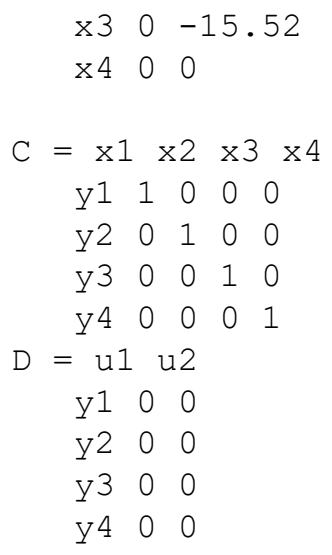

The system is assembled on command sys $=s s(A 0, B 0$, Cobs, Dobs $)$

The simulation result using the step (sys) function is shown in Fig. 4.

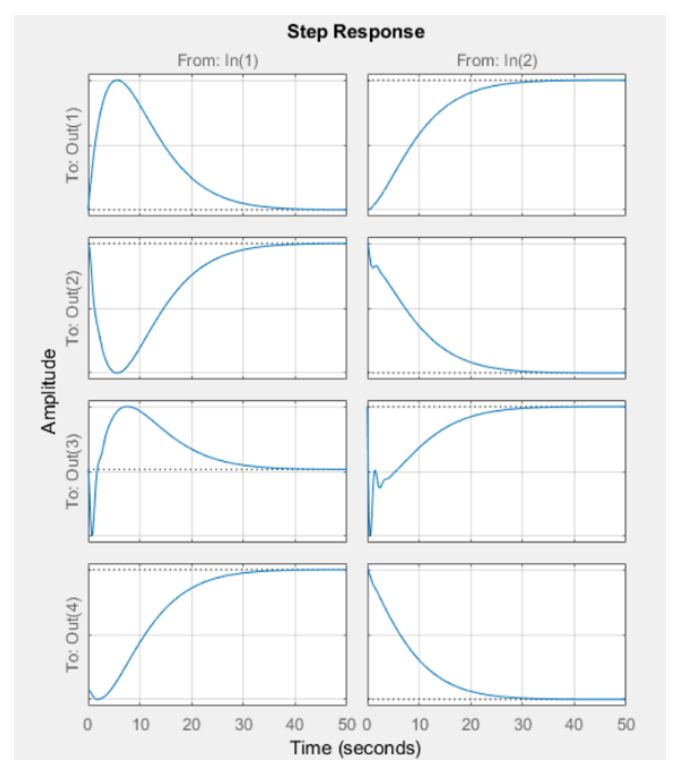

Fig. 4. The result of modeling a stabilization system without disturbances using the step (sys) function.

The model of disturbances due to wind loads in Simulink is as follows:

$$
\begin{array}{rl}
\text { W0 wind }=-1 & .0000 \quad 0 \\
0 & 0.0439 \\
0 & 0 \\
0 & 0
\end{array}
$$

Modeling of Gaussian white noise for wind (according to Kalman) is carried out according to the following program

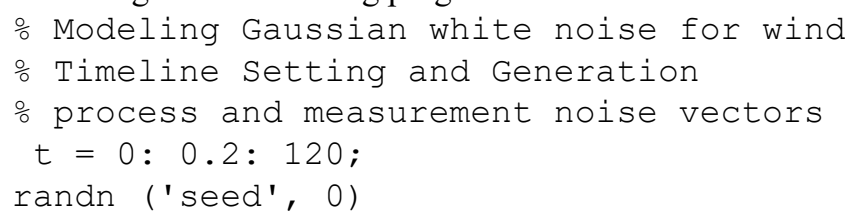




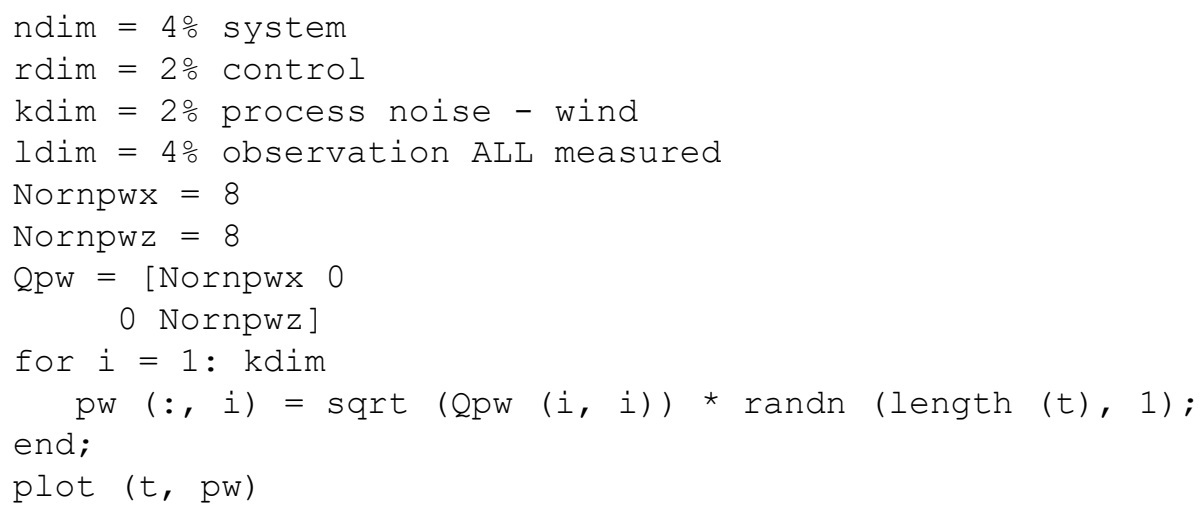

The result of disturbance modeling is shown in Fig. 5.

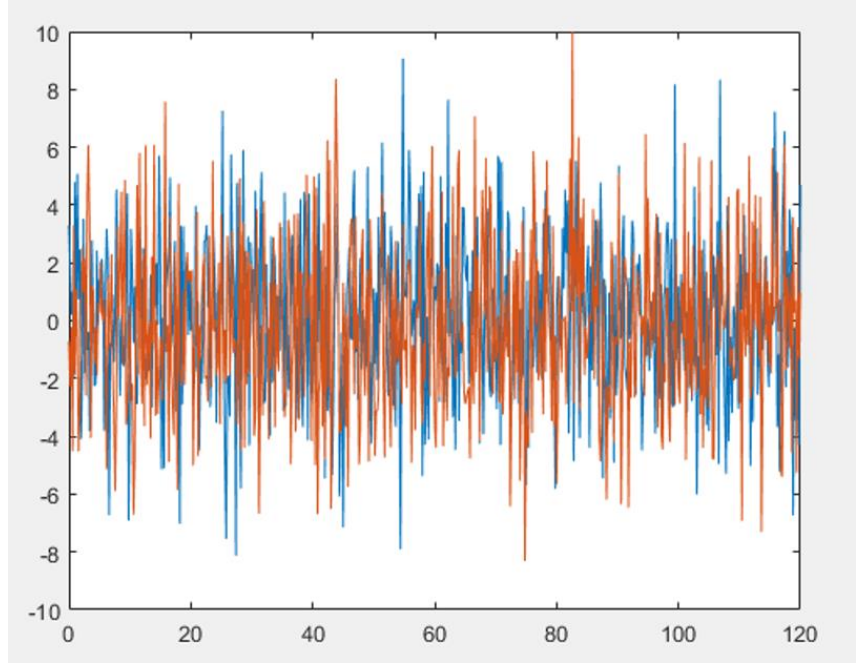

Fig. 5. The result of modeling wind load disturbances.

A model with process noise is calculated using the following code:

$\mathrm{X} 0 \mathrm{mod}=[\mathrm{XO}]$

Hobs $=\operatorname{zeros}($ ldim, $\mathrm{kdim})$

uwv = [u '; pwwing '] ';

Plantuwv = ss (A0, [B0, W0_wind], Cobs, [Dobs Hobs],

'inputname', \{'u_th u_ell pwx pwz'\}, 'outputname', 'y');

[youtuwv, tuwv, xuwv] $=1$ sim (Plantuwv, uwv, $t, x 0 m o d)$;

plot (tuwv, youtuwv $(:, 2,1)$ )

$\mathrm{X} 0 \mathrm{mod}=[\mathrm{X} 0]$

Hobs $=\operatorname{zeros}($ ldim, $\mathrm{kdim})$

uwv $=\left[\mathrm{u}^{\prime}\right.$ '; pwwing ']';

Plantuwv = ss (A0, [B0, W0_wind], Cobs, [Dobs Hobs],

'inputname', \{'u_th u_ell pwx pwz'\}, 'outputname', 'y');

[youtuwv, tuwv, xuwv] = lsim (Plantuwv, uwv, t, x0mod);

plot (tuwv, youtuwv $(:, 2,1)$ )

The simulation result of the stabilization system with process noise is shown in Fig. 6. 


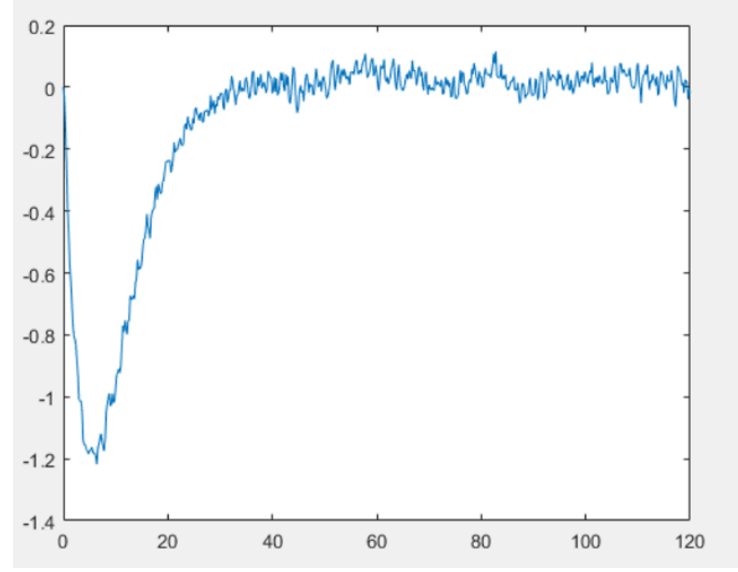

Fig. 6. The result of modeling a stabilization system with process noise.

The model with process noise and observation noise is calculated using the following code:

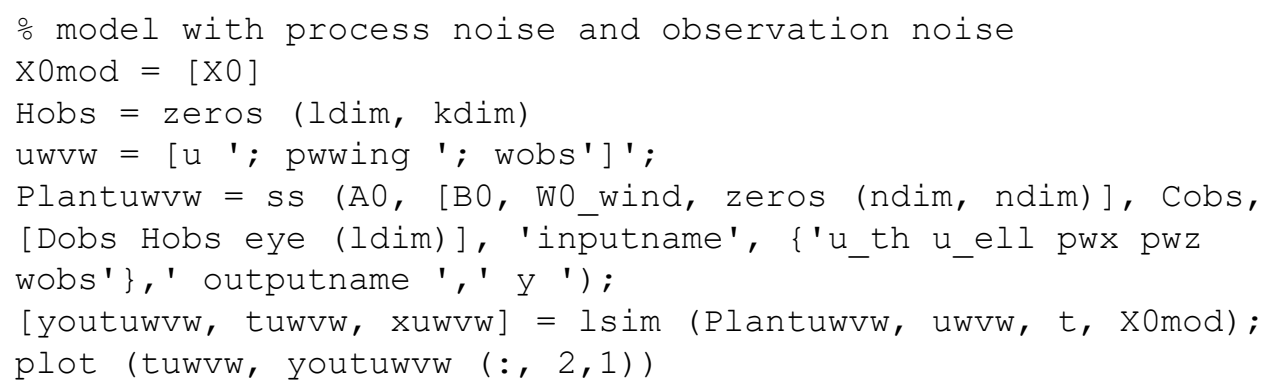

The simulation result with process noise and observation noise is shown in Fig. 7.

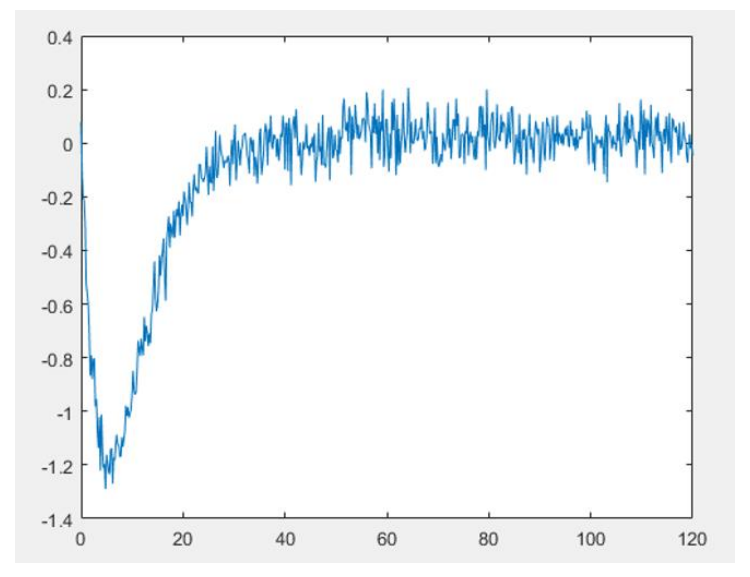

Fig. 7. Simulation result with process noise and observation noise.

A model with process noise and observation noise when applying harmonic influence (sine) to the input is calculated using the following code:

Tpersin $=\mathrm{pi}$ usin $=[$ zeros (length $(t), 1), \sin (t /$ Tpersin) $]$; plot (t, usin $(:, 2))$ 


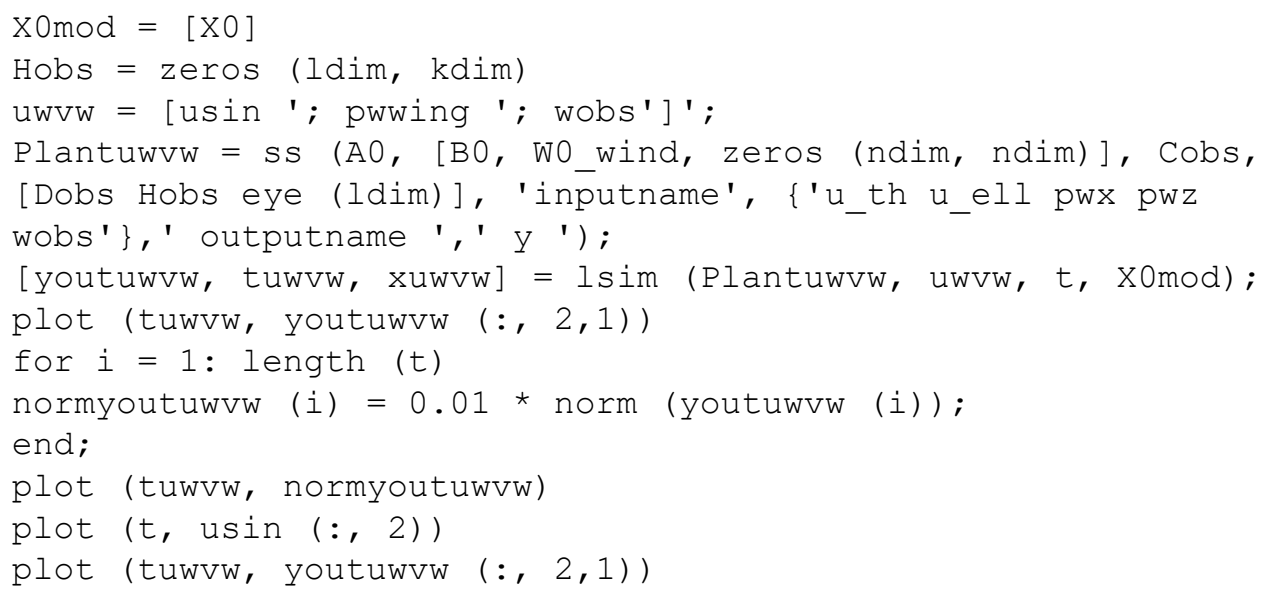

The result is shown in Fig. 8.

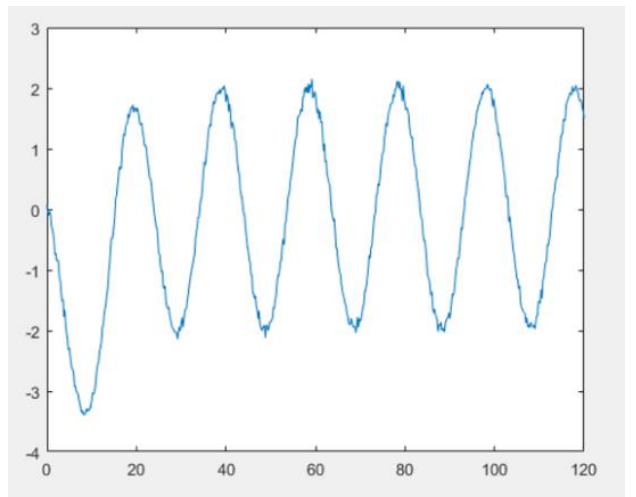

Fig. 8. Model with process noise and observation noise when applying harmonic input (sine).

The simulation results taking into account disturbances due to icing in Simulink are shown in Fig. 9, which shows the results of modeling the angle of attack when a sinusoidal effect is applied to the input for the absence of (data1) and the presence of icing (data2).

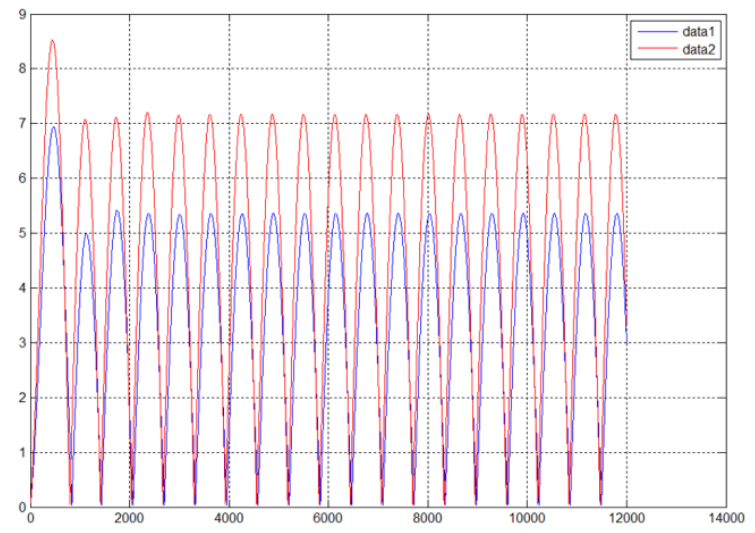

Fig. 9. Modeling the angle of attack when a sinusoidal effect is applied to the input for the absence of (data1) and the presence of icing (data2). 


\subsection{Comparative analysis of estimation and forecasting methods}

A sample of all possible combinations of all three factors is generated - the results of icing: a total of 1540 combinations. The technology of parallel computing in MATLAB is used (4 workers are involved). The simulation time was 30 minutes. A data ensemble is created to manage the simulation results, stored in the Data directory. In accordance with the principles of data mining, they are divided into training and test. The presence of icing to change the performance of Nuss sensors is shown in Fig. 10 and Fig. 11 in the form of histograms for the Nuss factor.

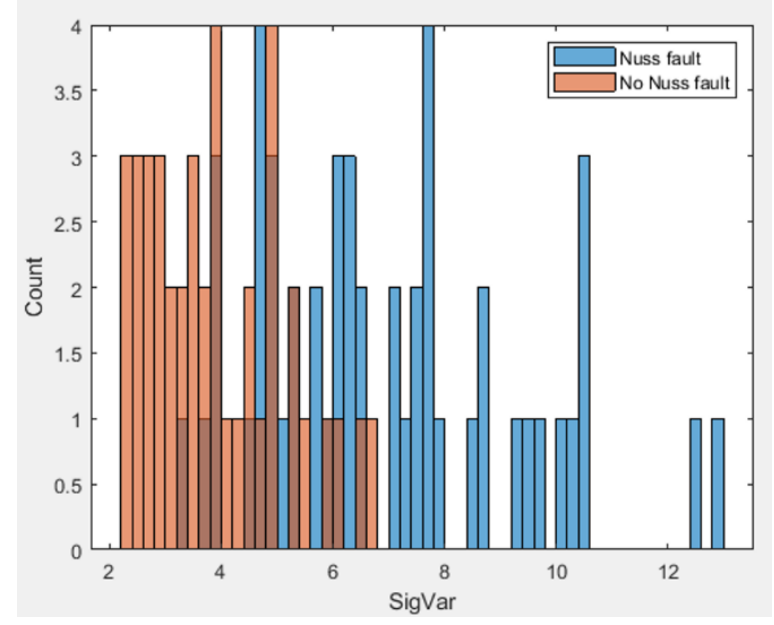

Fig. 10. Histograms for the Nuss factor (the effect of icing on changes in the performance of Nuss sensors) according to Var.

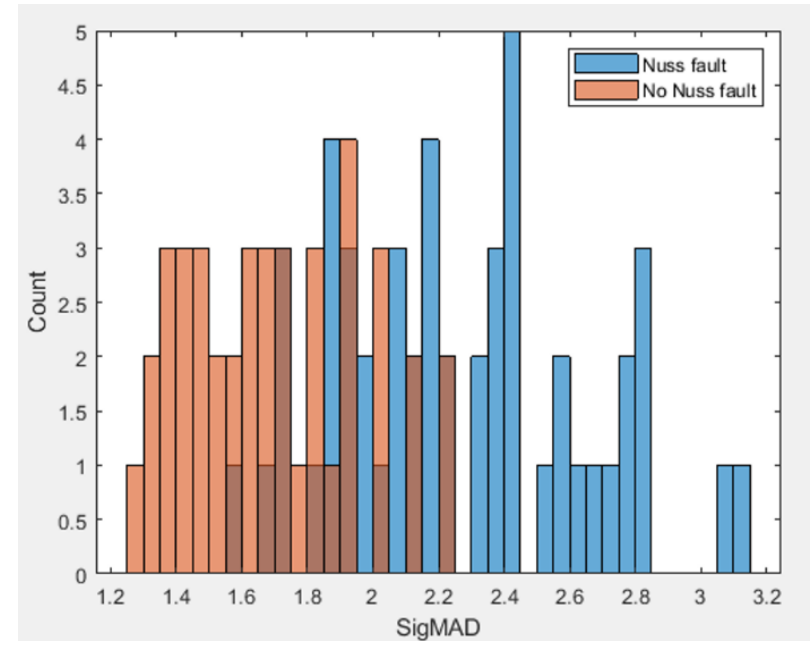

Fig. 11. Histograms for the Nuss factor (the effect of icing on changes in the performance of Nuss sensors) according to the MAD criterion.

It turned out that choosing the best predictor for the Nuss factor is to use Signal variance and Signal Median Absolute Deviation. 


\section{Conclusion}

The task of implementing rescue operations in the mountains is considered. A review of modern literature, including over the past two years. The main directions of research, as well as the problems and difficulties of practical implementation are identified. The specific task of assessing and predicting the influence of the factor of lowered temperatures and icing on the change in the models of the control object - UAVs, control means and distortion of the indicators of sensors is considered.

\section{References}

1. Yang Gao, Zhitao Lyu, Hamid Assilzadeh, Yang Jiang, Small and low-cost navigation system for UAV-based emergency disaster response applications, 4th Joint International Symposium on Deformation Monitoring (JISDM), 15-17 May 2019, Athens, Greece (2019)

2. Truong Duy Dinh, Rustam Pirmagomedov, Van Dai Pham, Aram A Ahmed, Ruslan Kirichek, Ruslan Glushakovand Andrei Vladyko1, Unmanned aerial system-assisted wilderness search and rescue mission, International Journal of Distributed Sensor Networks 2019, Vol. 15(5), The Author(s) (2019). DOI: 10.1177/1550147719850719. journals.sagepub.com/home/dsn

3. M.B. Bejiga, F. Mergani, A.A. Zeggada, Convolutional Neural Network Approach for Assisting Avalanche Search and Rescue Operations with UAV Imagery, Geology, Computer Science, Remote Sensing.2017, pp.693-696 (2017). DOI: 103390/rs9020100.

4. N.F.M. Salleh, W.M.W. Mohamed, R.E.M. Nasir, A.B.A. Mutaáli, S. Zainurin, Multirotor for Medical Aid Kit Transport, International Journal of Innovative Technology and Exploring Engineering (IJTTEE) ISSN: 2278-3075, Vol. 9, Issue-2, December (2019)

5. R.M. Carrillo-Larco, M. Moscoso-Porras, A. Taype-Rondan, A. Ruiz-Alejos and A. Bernabe-Ortiz, The use of unmanned aerial vehicles for health purposes: a systematic review of experimental studies, Global health, epidemiology and genomics (2020). https://www.cambridge.org/core. IP address: 91.193.177.127, on 18 Jan 2020.

6. Marzena Półka, Szymon Ptak, Łukasz Kuziora, The use of UAV's for search and rescue operations, TRANSCOM 2017: International scientific conference on sustainable, modern and safe transport, Procedia Engineering 192, pp.748 - 752 (2017)

7. Kyongsik Yun, Luan Nguyen, Tuan Nguyen, Doyoung Kim, Sarah Eldin, Alexander Huyen, Thomas Lu, Edward Chow, Small Target Detection for Search and Rescue Operations using Distributed Deep Learning and Synthetic Data Generation, p. 6 (2019)

8. Håkon B Abrahamsen, Use of an unmanned aerial vehicle to support situation assessment and decision-making in search and rescue operations in the mountains, Abrahamsen Scandinavian Journal of Trauma, Resuscitation and Emergency, Medicine, 22(Supp.1), p. 16 (2014). http://www.sjtrem.com/content/22/S1/P16

9. Abdallah Zeggada, Advanced classification methods for UAV Imagery, $\mathrm{PhD}$ International Doctorate School in Information and Communication Technologies, $\mathrm{p}$. 104 (2018)

10. Guilhem Bryant, An autonomous multi-UAV system for avalanche search, Master thesis July 3, p. 52 (2019) 
11. D. Giordan, A. Manconi, F. Remondino and F. Nex, Use of unmanned aerial vehicles in monitoring application and management of natural hazards, GEOMATICS, NATURAL HAZARDS AND RISK, Vol. 8, No. 1, p. 1-4 (2017).https://doi.org/10.1080/19475705.2017.1315619

12. Yunus Karaca, Mustafa Cicek, Ozgur Tatli, Aynur Sahin, Sinan Pasli, Muhammed Fatih Beser, Suleyman Turedi, The potential use of unmanned aircraft systems (drones) in mountain search and rescue operations, American Journal of Emergency Medicine 36, p. 583-588 (2018)

13. McCormack, Edward; Stimberis, John, Small unmanned aircraft evaluated for avalanche control, Transportation Research Record, no. 2169, pp. 168-173 (2010)

14. Vadim Kramar, UAS (drone) Arctic Challenges - Next Steps, Proceeding of the $25^{\text {th }}$ conference of fruct association, pp. 507-514. ISSN 2305-7254

15. SCAN TEAM REPORT NCHRP Project 20-68A, Scan 17-01. SUCCESSFUL APPROACHES FOR THE USE OF UNMANNED AERIAL SYSTEM BY SURFACE TRANSPORTATION AGENCIES.

16. Sonia Waharte and Niki Trigoni, Supporting Search and Rescue Operations with $U A V s$, p. 6 (2010). DOI: 10.1109/EST.2010.31

17. Mario Silvagni, Enrico Zenerino, Andrea Tonoli, Marcello Chiaberge, Multipurpose UAV for search and rescue operations in mountain avalanche events, Geomatics, Natural Hazards and Risk, October (2016). DOI: 10.1080/19475705.2016.1238852

18. N. N. Arefyev, Filter Kalman for solving the problem of coordinates UAV, Systemny analiz v informatika, No. 1.6, p. 26-34 (2019)

19. Gabriel M. Hoffmann and Steven L. Waslander, Distributed Cooperative Search using Information-Theoretic Costs for Particle Filters, with Quadrotor Applications AIAA Guidance, Navigation, and Control Conference and Exhibit, 21 - 24 August 2006, Keystone, Colorado (2006)

20. Zolt_an Beck, Collaborative Search and Rescue by Autonomous Robots, University of southampton, PhD. December 2016, p. 154 (2016)

21. Dmitriy Alexandrov, Elizaveta Pertseva, Ivan Berman, Igor Pantiukhin, Aleksandr Kapitonov, Analysis of Machine Learning Methods for Wildfire Security Monitoring with an Unmanned Aerial Vehicles,Proceeding of the $24^{\text {th }}$ conference of fruct association, pp. 3-9. ISSN 2305-7254

22. Mesay Belete Bejiga, Abdallah Zeggada, Abdelhamid Nouffidj and Farid Melgani, A Convolutional Neural Network Approach for Assisting Avalanche Search and Rescue Operations with UAV Imagery, Remote Sens, 9, 100; p. 22 (2017) doi:10.3390/rs9020100 www.mdpi.com/journal/remotesensing

23. Gheorghe Stancalie, A. Diamandi, A. Irimescu, V. Craciunescu, D. Mihailescu, O. Nicola, Rune Solberg, Arnt-Børre Salberg, Øivind Due Trier, M. Matreata, R. Mic, C. Corbus, M. Voiculescu, F. Ardelean, M. Török-Oance, Use of high resolution satellitebased products for snow monitoring and related domains in Romania, Outcomes and challenges of the SnowBall SEE project, Copernicus Pan European High Resolution Snow and Ice Monitoring Product -User consultation workshop Brussels, $7^{\text {th }}$ June (2018)

24. Irina Romanova, Metodologiya primeneniya intellektual'nogo analiza dannykh $v$ tekhnicheskikh prilozheniyakh, Tekhnologii razrabotki i otladki slozhnykh tekhnicheskikh system, Sbornik trudov V Vserossiyskoy nauchno-prakticheskoy konferentsii, pp.124-133 (2018) 
25. Ross D. Arnold, Hiroyuki Yamaguchi and Toshiyuki Tanaka, Search and rescue with autonomous flying robots through behavior-based cooperative intelligence Arnold et al, Journal of International Humanitarian Action 3:18, p. 18 (2018). https://doi.org/10.1186/s41018-018-0045-4

26. Christine M. Belcastro, Richard L. Newman, Joni K. Evans, David H. Klyde, Lawrence C. Barr, Ersin Ancel, Hazards Identification and Analysis for Unmanned Aircraft System Operations, AIAA AVIATION Forum. 5-9 June 2017, Denver, Colorado 17th AIAA Aviation Technology, Integration, and Operations Conference (2017)

27. Andrew Weinert, Scot Campbell, Adan Vela, Dieter Schuldt, and Joel Kurucar, WellClear Recommendation for Small Unmanned Aircraft Systems Based on Unmitigated Collision Risk, Journal of air transportation, Vol. 26, No. 3, July 2018, pp. 113-122 (2018). DOI: 10.2514/1.D0091

28. Samira Hayat, Evsen Yanmaz, Raheeb Muzaffar, Survay on Unmanned Aerial Vehicle Networks for Civil Applications, A communications Viewpoint, IEEE (2016)

29. Milan Erdelj and Enrico Natalizio, Kaushik R. Chowdhury, Ian F. Akyildiz, Help from the Sky: Leveraging UAVs for Disaster Management, Published by the IEEE CS $\mathrm{n}$ 1536-1268/17 (C) 2017 IEEE, pp. 24-32 (2017)

30. Milan Erdelj, Enrico Natalizio, Help from the Sky: Leveraging UAVs for Disaster Management, IEEE Pervasive Computing, January (2017). DOI: 10.1109/MPRV.2017.11

31. Arctic Search and Rescue Capabilities Survey. Enhancing international cooperation. 2017. Recommendations for an Interconnected Arctic. Report by the Arctic Economic Council's Telecommunications Infrastructure Working Group. Available online: https:// arcticeconomiccouncil.com/wp-content/uploads/2017/02/AEC-Report_FinalLR-1.pdf

32. Llugsi Ricardo, Escandón Renato, Implementation of a prototype for capture and digital processing of thermal images acquired from a UAV, Enfoque UTE, Vol.9, No.1, March 2018, pp. 1 - 11 (2018). http://ingenieria.ute.edu.ec/enfoqueute/ e-ISSN: 1390-6542 / p-ISSN: 1390-9363

33. Vsevolod V. Koryanov, Tatiana V. Kokuytseva, Alexey G. Toporkov, Stepan N. Iljukhin, Il'ya O. Akimov, Noa Mohamado, Victoria Da-Poian2, Concept development of control system for perspective unmanned aerial vehicles, MATEC Web of Conferences 151, 04010 (2018). https://doi.org/10.1051/matecconf/201815104010. ACMAE 2017

34. Mario Silvagni, Andrea Tonoli, Enrico Zenerino \& Marcello Chiaberge. Multipurpose UAV for search and rescue operations in mountain avalanche events, ISSN: 19475705 (Print) 1947-5713 (Online) Journal homepage: https://www.tandfonline.com/loi/tgnh20.

35. Eleftherios Lygouras, Nicholas Santavas, Anastasios Taitzoglou, Konstantinos Tarchanidis, Athanasios Mitropoulos and Antonios Gasteratos, Unsupervised Human Detection with an Embedded Vision System on a Fully Autonomous UAV for Search and Rescue Operations, Sensors 19, 3542; p. 20 p. (2019). DOI:10.3390/s19163542 www.mdpi.com/journal/sensors

36. Håkon B. Abrahamsen, A remotely piloted aircraft system in major incident management: concept and pilot, feasibility study, Abrahamsen BMC Emergency Medicine (2015) 15:12, p. 12 (2015). DOI 10.1186/s12873-015-0036-3 
37. Technical aspects of show avalanche risk management, Resources and Guidelines for Avalanche Practitioners in Canada, Canadian Avalanche Association (2016)

38. Randal W. Beard, Timithy W. McLain, Princeton University Press, Small Unmanned Aircraft: Theory and Practice (2012)

39. I.K. Romanova, Metody sinteza sistemy upravleniya letatel'nymi apparatami, Moskva, Izd. BMSTU (2017)

40. Mathworks. Predictive Maintenance Toolbox ${ }^{\circledR}(2019)$ 